\title{
Petascale Cloud Supercomputing for Terapixel Visualization of a Digital Twin
}

\author{
Nicolas S. Holliman, Member IEEE Computer Society, Manu Antony, \\ James Charlton, Stephen Dowsland, Philip James and Mark Turner
}

\begin{abstract}
Background-Photo-realistic terapixel visualization is computationally intensive and to date there have been no such visualizations of urban digital twins, the few terapixel visualizations that exist have looked towards space rather than earth. Objective-our aims are: creating a scalable cloud supercomputer software architecture for visualization; a photo-realistic terapixel 3D visualization of urban IoT data supporting daily updates; a rigorous evaluation of cloud supercomputing for our application. Method-We migrated the Blender Cycles path tracer to the public cloud within a new software framework designed to scale to petaFLOP performance. Results-we demonstrate we can compute a terapixel visualization in under one hour, the system scaling at $98 \%$ efficiency to use 1024 public cloud GPU nodes delivering 14 petaFLOPS. The resulting terapixel image supports interactive browsing of the city and its data at a wide range of sensing scales. Conclusion-The GPU compute resource available in the cloud is greater than anything available on our national supercomputers providing access to globally competitive resources. The direct financial cost of access, compared to procuring and running these systems, was low. The indirect cost, in overcoming teething issues with cloud software development, should reduce significantly over time.
\end{abstract}

Index Terms—Data Visualization, Internet of Things, Scalability, Supercomputers

\section{INTRODUCTION}

A $S$ we gather increasing amounts of data about our Aurban environment it is important to present this in informative, engaging and accessible ways so that the widest possible set of stakeholders have the potential to see the data. The Newcastle Urban Observatory [1] has been collecting loT sensed environmental data about the city of Newcastle-upon-Tyne for over three years, gathering more than one billion data records to date. As is common to many data platforms the rate of data collection is significantly faster than the rate at which humans can comprehend and learn from the information the data carries [2]. Therefore, we explore how we can present descriptive statistics, such as hourly sensor averages, in a realistic 3D visualization of the city and do so at a range of geographic scales.

Terapixel images are images that contain over one trillion pixels and, within the right toolset [3], provide an in-

- N.S. Holliman is with the School of Computing, Newcastle University, Newcastle-upon-Tyne, NE4 5TG. E-mail:

nick.holliman@newcastle.ac.uk

- M. Antony is with the School of Computing, Newcastle University, Newcastle-upon-Tyne, NE4 5TG.E-mail:m.antony@newcastle.ac.uk

- J. Charlton is with the Department of Architecture and Built Environment, Northumbria University, Newcastle-upon-Tyne, NE1 8ST. Email:j.charlton@northumbria.ac.uk

- S. Dowsland is with the School of Computing, Newcastle University, Newcastle-upon-Tyne, NE4 5TG. E-mail: stephen.dowsland@newcastle.ac.uk

- P. James is with the School of Engineering, Newcastle University, Newcastle-upon-Tyne, NE4 5TG. E-mail: philip.james@newcastle.ac.uk

- M. Turner is with the School of Computing, Newcastle University, Newcastle-upon-Tyne, NE4 5TG. E-mail: mark.turner@newcastle.ac.uk tuitive, fluid user experience where the viewer can see an overview of the whole image or zoom into incredible detail. To the best of our knowledge we present here the first terapixel visualization of IoT data within a 3D urban environment. To visualize the city and its data we have chosen an advanced path-tracing renderer that is more typically used for cinematic and architectural rendering. We selected Cycles, from the Blender toolset [4], because of its high quality physically based lighting simulation calculations.

The combination of high-quality rendering and terapixel imaging is an attractive one for users and allows us to explore new ways of visualizing urban IOT data within its city context. However, while the end user experience is compelling there is a significant computational cost to producing a high quality terapixel image. To solve this challenge, we investigate the use of petaFLOP supercomputer systems in the Azure cloud at a scale not previously reported. We address three research questions:

Scalability: how to design a new supercomputer application architecture, larger than any system available in the UK, for scalable visualization using the public cloud?

Visualization: can the cloud help render the first terapixel urban loT visualization supporting live daily updates?

Evaluation: the rigorous performance evaluation of cloud supercomputing for visualization applications.

\section{BACKGROUND}

We review three background topics: urban data visualization, scalable computing in the cloud, and existing architectures for distributed and cloud rendering. 


\subsection{Urban Data Visualization}

Urban data visualization brings together large 3D models of cities and countries with real time and historical data from many sources including loT sensing devices [5]. The concept of a digital twin for observing, exploring and predicting urban behavior is rapidly gaining ground [6]. Visualization methods directly support the Gemini principle [7] of creating insight into the data in a digital twin.

The Royal Society [8] proposes that for data to be trustworthy it needs to be made intelligently open, in particular, to be accessible, intelligible and assessable. Our investigation of terapixel imaging explores how the accessiblity and intelligibilty of urban loT data can be improved, potentially leading to better assessability of its reliablity, meaning and value. To achieve this our terapixel 3D urban visualization has five goals [9] aiming to be:

Truthful. to show accurate statistical data about the city, to scale and situated within its spatial context.

Functional. to create an interactive scalable visualization that works across platforms with low overhead.

Beautifut. to use the highest quality rendering tools, applying cinematic quality production techniques.

Insightful. to reveal new insights across scale about the urban environment in the city of Newcastle-upon-Tyne.

Enlightening. accessible to the viewer so they are able to gain a deep comprehension of the data.

Terapixel images have been previously used to visualize astronomical observations [10] and multiple gigapixel images have been used for visualizing cosmology simulation outputs [11][12]. We aim to apply terapixel imaging to look towards, instead of away from, Earth and to present observations about one region on Earth in detail.

\subsection{Scalable Cloud Computing}

Public cloud services have started to offer as laaS (Infrastructure as a Service) an increasing amount of GPU compute capacity, this enables supercomputer scale compute performance to be deployed, utilised and released ondemand. Current examples include the Azure N-series [13] and AWS EC2 Elastic GPUs [14]. How far cloud systems can scale is a question of some current interest [15].

In the cloud, as with any parallel system, the same classical laws bound a system's scalability. Amdahl's law [16] can be summarized as: any one parallel computation is limited in scalability as the number of nodes $n$ increases by the fraction of the problem that cannot be parallelized. If $p$ is the parallel fraction, and $f$ the serial fraction, then speedup $S$ is given by:

$$
S(n)=\frac{1}{f+\frac{p}{n}}
$$

We need to take care using the cloud that additional management services don't add undue serial overhead and limit our performance. Even at $f=0.1 \%$ the overhead will limit our best speedup to 506 for $n=1024$ nodes.

The Gustafson-Barsis' law [17] reconsidered how parallel computers are used in practice and defined scalability in relation to problem size, arguing that the problem size is always scaled to fill the compute capacity:

$$
S(n)=n-f(n-1)
$$

In the rendering approach we have chosen here the problem can scale to fill the largest machine we have available. As a result, we would expect to see the Gustafson-Barsis law holding if the problem size remains large enough. We should therefore see efficient linear scaling as we add more compute nodes. Even so there is a limit to the compute size of our problem and at that point Amdhal's law will ultimately define our scaling limit.

In later work Gustafson [18] argued the for use of ends based performance measures as well as pure computational metrics. As our interest here is application driven as much as system driven, we also consider the following from Gustafson's proposed ends-based metrics:

- Time to compute the answer.

- Completeness of the answer.

- Maximum feasible problem size.

- System reliability.

- Performance divided by the system cost.

We also add an additional application metric, future scaling, which provides a prediction of how far into the future it will be before the system performance we achieve on a supercomputer today might be available on everyday desktop workstations.

\subsection{High Performance Visualization Systems}

Many distributed and parallel rendering system architectures for High Performance Visualization (HPV) have been proposed as new High Performance (HPC) and High Throughput (HTC) platforms have appeared [2].

From the earliest days of HPV systems, rendering has often been thought of as ideally parallel even though this is not the case when geometry data sizes exceed local memory or network bandwidth [19]. Nonetheless, rendering is a good test of new hardware performance since it is capable of scaling to follow Gustafson's law, absorbing all available compute resource. This is particularly the case for physically based path tracing algorithms whose performance is dominated by floating point calculations.

Remote HPV systems can be categorised as being send-data, send-geometry or send-image [2]. Send-data systems assume a local GPU at the client and the server sends a subset of the application data to be converted to geometry and rendered locally into an image. Sendgeometry systems convert the data to geometry at the server and send just geometric and material data to the client to be rendered. In both cases the client needs substantial network, and compute resources, and in the worst case as the data size grows the client-side resource demand is unbounded [20].

The third approach send-image, and the one we take in this project, is where the geometry is generated and rendered on the server and only the image is sent to the client. The key advantage is the maximum image data required at any one time is bounded by the fixed number of pixels on display rather than the unbounded data or model geometry. This bounds the network bandwidth needed between server and client. 
A similar approach to ours was described by Chen et al in [21] which generalises previous image-based rendering systems such as QuickTime VR. Chen uses a multiresolution tiled image as the output format and serves this from an http server to viewers running in client browsers. Our work differs in that we aim to use the public cloud to render the terapixel images and to parallelize all the operations needed to output the multiresolution tiled image format in addition to the rendering operations.

While GPU solutions are in widespread use Intel have recently argued [22] that software (CPU) only solutions such as OSPRay have advantages for exascale data sets that do not fit in high speed GPU memory. This GPU-CPU tradeoff depends on whether data sets are too big to move and whether they can be subsetted for rendering in GPU memory, in our case we can hold the model data in GPU memory and GPU retains a performance advantage.

One solution to our visualization problem could be to use a render farm, both private and public render farms exist and render services are available in the cloud. However, currently these don't offer all the functionality we need to build an end-to-end terapixel solution, they are primarily tuned to production animation rendering.

\subsection{Summary}

We aim to generate high quality, terapixel urban data visualizations that are rendered by a system that scales to efficiently use as many compute nodes as are available. We have chosen to design this as a send-image system architecture so that thin-clients are as capable of displaying the visualization as fat-clients. Aiming to support wide accessibility to our advanced visualization results.

\section{Visualization System Architecture}

We have previously discussed [20] how a send-image architecture for remote cloud rendering, where the rendering is server-based and a pixel-stream is sent to the client, has important advantages including 1) Decoupling the task of display from the computationally complex task of rendering, enabling the use of thin-clients to view complex visualizations, 2) Limiting the maximum bandwidth needed to the client display device, which is otherwise unbounded in fat-client solutions. The following details the requirements and the architecture of such a system for terapixel visualization.

\subsection{The Visualization}

As shown in Fig. 1 we used a $1.28 \mathrm{~km} \times 1.28 \mathrm{~km}$ 3D map of central Newcastle-upon-Tyne as the context for our visualization. This defined the geographic boundaries of the visualization and the loT sensors we included. Since a terapixel image consists of $2^{20} * 2^{20}$ or $(1,048,576)^{2}$ pixels this means that one pixel in our terapixel image represents approximately $1.2 \mathrm{~mm} \times 1.2 \mathrm{~mm}$ in the real world. This provides the ability in a single image to zoom in from city scale to an area of about the size of desk in a room in a building. This represents a zoom factor of approximately $512 x$ on a full HD display allowing users to view data in the city at a wide range of scales.

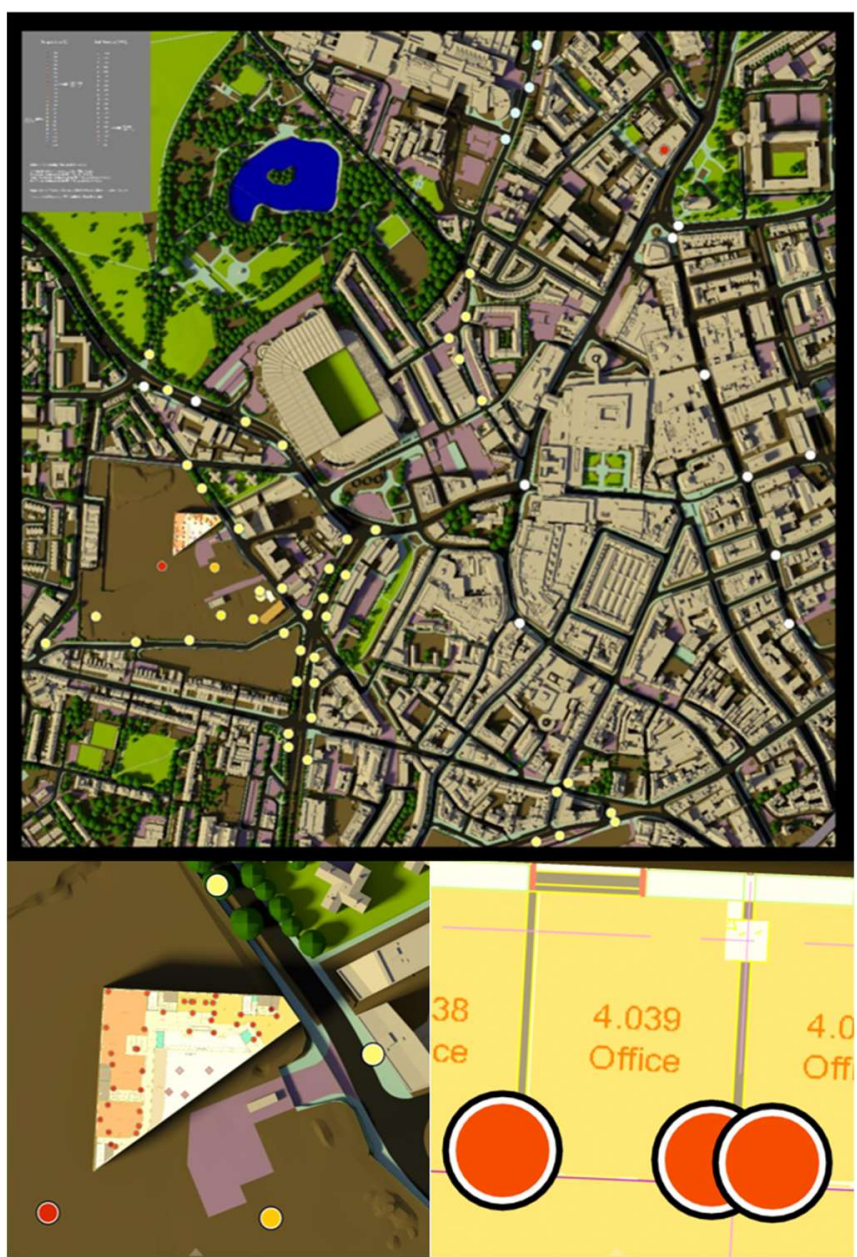

Fig. 1. The terascope image of Newcastle-upon-Tyne, the inset images are zoomed in views illustrating the internal sensors in the Urban Sciences Building, glyph colours represent the average temperature at each sensor over one hour, glyphs are rescaled as the viewer zooms in so that they retain a reasonable size in the image.

Set within this map are glyphs representing the location of Urban Observatory loT sensors [1] around the city, the sensors are situated both externally in the city and internally within the Urban Sciences Building. For the purposes of this visualization we use an arbitrary day's temperature values, averaged over one hour for each of the sensors. The average temperature value is represented as a solid colour at the centre of each glyph, though not obvious in a single still image the glyphs automatically orient themselves to face the camera and rescale at preset zoom levels to maintain a reasonable size. Some of the glyphs represent sensors that are physically as close as $100 \mathrm{~mm}$ in the real world.

\subsection{Technical Requirements}

One terapixel image requires approximately one terabyte of storage, something that is not currently easy to download as a single image file to most clients. Instead, we manage image viewing as a streaming process using a client-side image streaming tool, the krpano Viewer [3]. This requires the image to be stored on the server in a hierarchy, with each layer of the pyramid a complete im- 
TABLE 1

TERAPIXEL IMAGE PROPERTIES

\begin{tabular}{rrrrr}
\hline $\begin{array}{r}\text { Pyramid } \\
\text { level }\end{array}$ & $\begin{array}{r}\text { Image side } \\
\text { length } \\
\text { (pixels) }\end{array}$ & $\begin{array}{r}\text { Total number of } \\
\text { pixels at this } \\
\text { level }\end{array}$ & $\begin{array}{r}\text { Number of } \\
512 \times 512 \\
\text { tiles }\end{array}$ & $\begin{array}{r}\text { Tile side } \\
\text { length in } \\
\text { the real } \\
\text { world } \\
(\mathbf{m m})\end{array}$ \\
\hline \hline $\mathbf{1 2}$ & $\mathbf{1 0 4 8 5 7 6}$ & $\mathbf{1 0 9 9 5 1 1 6 2 7 7 7 6}$ & $\mathbf{4 1 9 4 3 0 4}$ & $\mathbf{6 2 5}$ \\
11 & 524288 & 274877906944 & 1048576 & 1250 \\
10 & 262144 & 68719476736 & 262144 & 2500 \\
9 & 131072 & 17179869184 & 65536 & 5000 \\
$\mathbf{8}$ & $\mathbf{6 5 5 3 6}$ & $\mathbf{4 2 9 4 9 6 7 2 9 6}$ & $\mathbf{1 6 3 8 4}$ & $\mathbf{1 0 0 0 0}$ \\
$\mathbf{7}$ & 32768 & 1073741824 & 4096 & 20000 \\
6 & 16384 & 268435456 & 1024 & 40000 \\
5 & 8192 & 67108864 & 256 & 80000 \\
$\mathbf{4}$ & $\mathbf{4 0 9 6}$ & $\mathbf{1 6 7 7 7 2 1 6}$ & $\mathbf{6 4}$ & $\mathbf{1 6 0 0 0 0}$ \\
3 & 2048 & 4194304 & 16 & 320000 \\
2 & 1024 & 1048576 & 4 & 640000 \\
1 & 512 & 262144 & 1 & 1280000 \\
\hline \hline
\end{tabular}

The terapixel image is a hierarchical pyramid of $512 \times 512$ pixel tiles, this supports interactive panning and zooming for the image.

age, tiled into $512 \times 512$ image tiles. The properties of each level in the pyramid are shown in Table 1 where the side length of each layer doubles with depth in the pyramid.

To create the image pyramid, we could render each level individually or render just the highest resolution level 12 and sub-sample this to calculate all the lower levels in the hierarchy. However, we adopt a compromise where we render levels 12, 8 and 4 and subsample these levels to fill in each set of three intermediate levels. This heuristic saves total rendering time compared to rendering every level, and reduces compression errors in the lower level images compared to repeatedly subsampling the full image. This approach also allows us to render features such as the loT sensor glyphs at different scales in the image for the three key hierarchy levels, keeping the glyphs a more appropriate size as the viewer zooms the image. The choice to render a level could be automated by setting maximum PSNR threshold differences between levels.

To calculate intermediate levels in the image pyramid from rendered levels we can apply a general smoothing function [23]. Any layer / 1 can be generated from a higher resolution level / by sub-sampling using a kernel weighted filter such that a sub-sampled pixel $G_{l-1}(i, j)$ is related to the pixels in a higher resolution image by:

$$
G_{l-1}(i, j)=\sum_{m} \sum_{n} w(m, n) G_{l}(2 i+m, 2 j+n)
$$

where the weighting function $w(m, n)$ is chosen to balance between the speed of computation and quality of the sub-sampled layers.

\subsection{Design}

The computation we need to undertake is now defined. To generate a one terapixel image we need to compute an image pyramid of 5,592,405 image tiles. Every fourth level in the pyramid will be rendered directly and the remaining intermediate levels will be computed by subsampling the rendered layers.

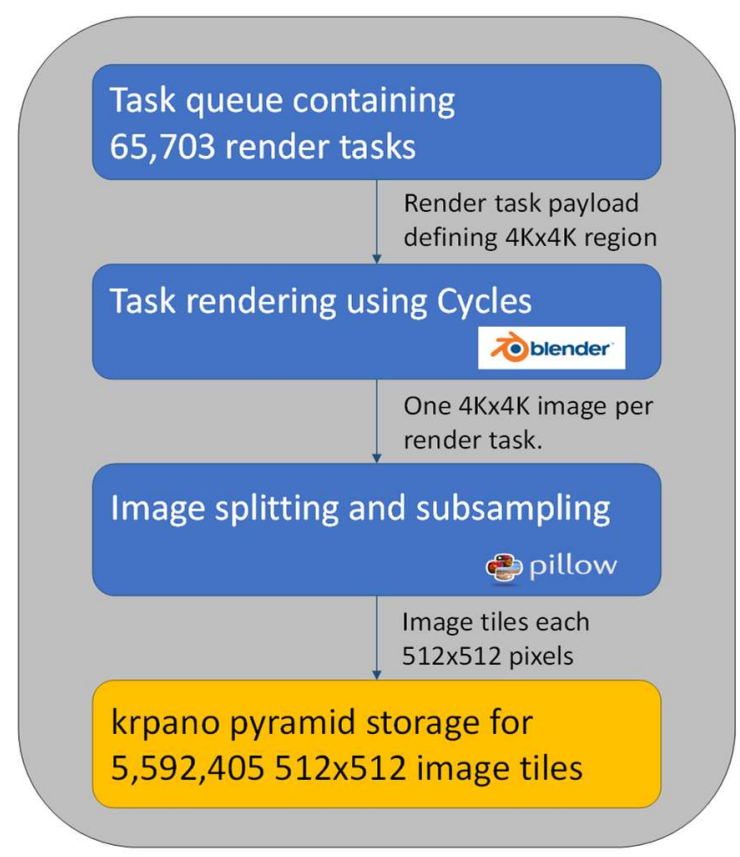

Fig. 2. Overview of the data flow for the compute tasks required to generate a single terapixel image pyramid. Blender is used to render the images and Pillow generates the krpano image tiles.

As shown in Fig. 2 we group image tiles for rendering efficiency render into tasks of 4096×4096 pixels which are then split and subsampled as required into tiles. We therefore need to compute a total of 65,703 render tasks. For the example here, our entire 3D city model is assumed to be replicable to every compute node while each task definition has a relatively small payload defining the $4 \mathrm{~K} \times 4 \mathrm{~K}(\mathrm{~K}=1024)$ region to be rendered. The result is an almost ideally task parallel application, with little communication overhead compared to compute demand. This makes it suitable as a starting point for testing the scalability of cloud supercomputing.

\section{SySTEM DESIGN AND IMPLEMENTATION}

In order to implement a highly scalable parallel version of the data flow architecture in Fig. 2 we used a combination of Microsoft Azure tools and our own system software.

\subsection{Scalable public cloud framework}

We previously implemented a rendering framework for urban loT data that was designed to update images from live IoT data using a mixture of public and private cloud resources [24]. This was capable of rendering images up to UHD $(3840 \times 1920)$ resolution and was used to update images of loT data every few minutes throughout the day.

The size of the computational task for the terapixel image was too large for our private cloud. We therefore created the terapixel system using public cloud components. To create a scalable framework for the computation we used the following components as shown in Fig. 3:

- We inject the 65,793 rendering tasks into an Azure Batch task queue and allow the queue to distribute the tasks between the pool of compute nodes. It also manages node failure and replacement. The tasks are 
added to the queue using Azure Functions, a serverless service that runs single scripts on-demand. The script creates three separate jobs, one each for levels 4,8 and 12 in the image pyramid, and then creates the render tasks for each job. Each task contains the data required to start Blender when run on a node. Tasks are added in batches of 64 using the addCollection method to reduce the total number of http calls over adding each task individually. However, addCollection does not guarantee tasks are accepted by the queue. An array of failed submissions is kept and used as input to call the same function recursively until there are no failed task submissions.

- Compute nodes are packaged as an Azure Managed Image that uses Python to run Blender with the whole city model and Pillow to split the images into tiles. A pool of compute nodes is then requested manually, using the managed image as a template. Using a managed image over Azure Batch Start Task inputs reduces the cumlative setup time of each node across each render. The number of compute nodes is varied to adjust for the performance data collection. Note that compute nodes can, and do, fail during execution and Azure should seamlessly swap these back in.

- Azure Blob Storage provides a scalable solution to storing the 5,592,405 output image tiles in a folder hierarchy suitable for the krpano viewing tool. By default, Blob Storage has an http server interface suitable for serving the client-side krpano app with the image tiles it requests when a user views the image.

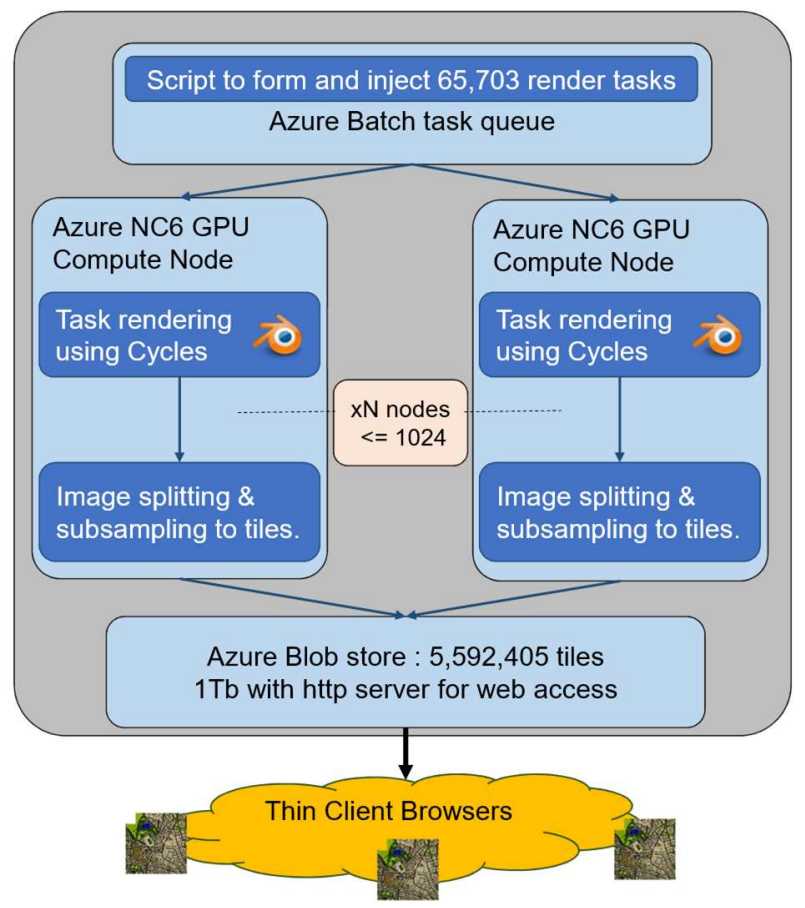

Fig. 3. Cloud supercomputing system architecture constructed using Azure ${ }^{\mathrm{TM}}$ public cloud laaS including Azure Batch task queue and Azure NC6 Compute nodes, Azure infrastructure is shown in light blue, other colours highlight specialist code for our computation.
While the batch queue and task distribution were managed by Azure we still had to run custom health checks on the nodes to ensure that drivers versions were correct and that the GPU was still accessible to the Blender. On some occasions we found that driver issues arose that required action to update and reboot the machine. Healthchecks and required actions were carried out using Ansible [25]. Ansible is an IT automation engine, which allowed us to run arbitrary commands across our batch pool through an ssh connection. Ansible requires the creation of a node inventory in order to connect to a pool of machines. This inventory was generated using the Azure Batch API [26].

\subsection{Blender Cycles Rendering}

Blender Cycles is an unbiased physically based path tracing engine which creates an image by tracing light rays backwards from the camera through a scene. Because it simulates realistic effects such as soft shadows, caustics and glossy surfaces it can require very significant compute times. However, also because it supports these effects it allows the use of advanced cinematic visualization effects in an urban smart city visualization.

TABLE 2

TERAPIXEL TASK PROPERTIES

\begin{tabular}{|c|c|c|c|}
\hline $\begin{array}{l}\text { Hierarchy } \\
\text { level }\end{array}$ & $\begin{array}{l}4 \mathrm{~K} \times 4 \mathrm{~K} \text { pixel image } \\
\text { rendering tasks }\end{array}$ & $\begin{array}{l}512 \times 512 \\
\text { pixel output } \\
\text { tiles }\end{array}$ & $\begin{array}{l}\text { Estimated storage } \\
\text { for JPEG coded } \\
\text { tiles in kB }\end{array}$ \\
\hline 12 & 65536 & 4194304 & 436207616 \\
\hline 11 & & 1048576 & 109051904 \\
\hline 10 & & 262144 & 27262976 \\
\hline 9 & & 65536 & 6815744 \\
\hline 8 & 256 & 16384 & 1703936 \\
\hline 7 & & 4096 & 425984 \\
\hline 6 & & 1024 & 106496 \\
\hline 5 & & 256 & 26624 \\
\hline 4 & 1 & 64 & 6656 \\
\hline 3 & & 16 & 1664 \\
\hline 2 & & 4 & 416 \\
\hline 1 & & 1 & 104 \\
\hline Totals & 65,793 & $5,592,405$ & $581,610,120$ \\
\hline
\end{tabular}

One terapixel image requires 65,793 task computations generating $5,592,405$ image tiles in 12 hierarchical levels requiring approximately $0.54 \mathrm{tB}$ of Azure Blob ${ }^{\mathrm{TM}}$ storage, levels 4,8 and 12 are computed, other levels are downsampled from these results.

There are a considerable number of optimisation and tuning parameters in Blender Cycles, and we ran a series of trials with the aim being to optimize image quality and compute time. For GPU supported execution we set the number of samples per pixel to be 20, the Cycles tile size to be $256 \times 256$ and the post-rendering image denoising radius to be 5 . This resulted in a single $4 \mathrm{~K} \times 4 \mathrm{~K}$ task rendering time of approximately 150 seconds on the target hardware described below.

\subsection{Pillow for Image Splitting and Sub-sampling}

The output from Blender for each render task is a $4 \mathrm{kx} 4 \mathrm{k}$ image and this needs to be split into $512 \times 512$ tiles and subsampled to form the tiles for the three lower resolution layers beneath each rendered layer in the pyramid, as described in Table 2. 
To retain image quality for the splitting process Blender is requested to output a lossless PNG format image. This is then passed to a python script calling the Pillow API which both splits the $4 \mathrm{~K} \times 4 \mathrm{~K}$ image into $512 \times 512$ tiles and subsamples it to form the lower resolution layers using the resize function. All 512×512 tiles are stored using JPEG to save storage space and client transfer bandwidth.

\subsection{Performance metric collection}

To understand the performance of the system we designed into the architecture a series of performance metric data collection points. These generate typed, timed messages at key points in the computation, for example rendering start and end points, that are stored in a dedicated database. Table 3 summarises the subset of metrics we collect that are most relevant to this article.

TABLE 3

TeRAscope PeRformance Metrics

\begin{tabular}{lll}
\hline Metric & Units & Frequency \\
\hline \hline GPU Utilization & percentage & $1 \mathrm{~Hz}$ \\
GPU Temperature & degrees Centigrade & $1 \mathrm{~Hz}$ \\
GPU Power & Watts & $1 \mathrm{~Hz}$ \\
Render duration & seconds & per task \\
Tiling duration & seconds & per task \\
Storage duration & seconds & per task \\
\hline \hline
\end{tabular}

During each run of the system we collect a continuous stream of data from all the operational compute nodes in addition to Azure's

standard metrics, the most relevant subset is shown here.

The performance metric capture system is built on software commonly refered to as the ELK stack, ELK being a combination of Elasticsearch, Logstash and Kibana. Elasticsearch is a distributed, RESTful search and analytics engine [27]. Logstash is a data processing pipeline that ingests data from multiple sources, transforms it, and sends it on to a storage system. Kibana is a visualization tool for exploring data stored in Elasticsearch.

Data stored in Elasticsearch is arranged into indexes. Indexes are collections of data points that share the same data model. The Terascope performance metrics use three different indexes; render, GPU and Metricbeat. All the data stored in each of these indexes is time series records of the form: value of metric $X$ at time $Y$ was $Z$. The render index stores all the render events as an image is generated on the node. The GPU index stores all the performance metrics that can be captured from the GPU hardware for every node in the entire pool. GPU data is captured by Logstash every second and sent to the GPU index. Finally, the Metricbeat index is populated using a Logstash tool called Metricbeat. Metricbeat captures data about the state of all the hardware (except for the GPU) on each of the nodes. There are many granular details to this data but broadly it captures usage information about all the CPU cores, memory, disks and networking.

These metrics complement the metrics generated by Azure which don't record at the same level of granularity. This approach also allowed us to compare our own measures of run time with Azure's measures providing a way to track any overhead from the Azure framework.

The total runtime system load for our metrics collection is not significant compared to the overall compute load and each full terapixel image run generates around 65 Gigabytes of performance data. However, we did need to ensure we had enough server disk bandwidth to handle the flow of metric messages on the largest system tested.

\section{Evaluation}

\subsection{Choosing a cloud virtual machine}

Current public cloud offerings present a range of predefined virtual machine specifications to choose from and a number of possible global locations. For the compute nodes in the first set of evaluation runs reported here we choose a lower end virtual machine to control our costs, the Azure NC6 node. This consists of an Intel Xeon E52690v3 CPU, a K80 NVIDIA GPU with 56Gbytes of memory and 380 Gbytes of SSD disk space. Each NC6 virtual machine however shares the CPU and the GPU with a second virtual machine and has half the CPU cores and one half of the K80 GPU card. This results in each NC6 node having six CPU cores and one GPU. Therefore, the theoretical GPU compute performance of each NC6 node is approximately 2.8TFLOPS in single precision mode [28] which is the mode used by Blender. The largest pool size we requested was $128 \mathrm{GPU}$ NC6 nodes, this provided a theoretical peak performance of 0.36 PFLOPS.

In addition, we use Azure resources to organize the computation: one node for running the task queue and one terabyte of Azure blob storage for the image tiles making up the terapixel image. An additional node hosts an Elasticsearch database that is used for storing all the performance metric data generated during each run.

\subsection{System scaling pilot}

To pilot the scaling performance of the system we undertook two evaluations; an initial series of runs generating a gigapixel image (32678×32768 pixels) and a second computing the full terapixel image (1048576x1048576 pixels).

The gigapixel test was used to evaluate scaling over the full range of compute nodes from 1 to 128 nodes. However, as the gigapixel image consists of just 256 render tasks we predicted a drop off in scaling efficiency with higher numbers of compute nodes. This was because the variation in run time between tasks means the number of tasks available per node could drop to less than two for some nodes and as a result at the end of the computation some nodes are left idle for significant amounts of time.

The gigapixel results are summarised in Fig. 4. The system scales reasonably, although sub-linearly, up to 96 nodes. Then the scaling efficiency reduces markedly as predicted, down to $63 \%$ of expected speedup at 112 and 128 nodes. 


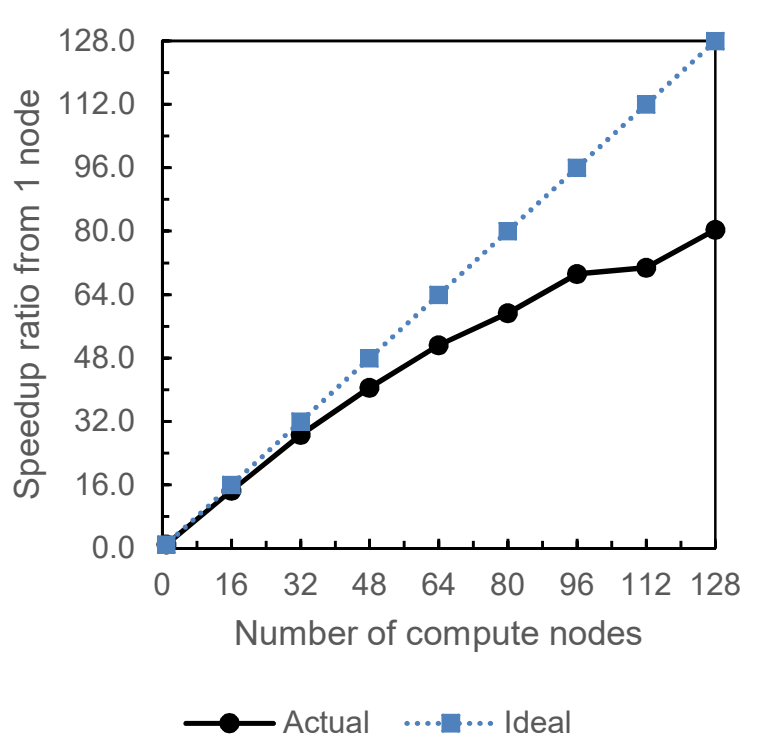

Fig. 4. Evaluating the scalability of the gigapixel computation on NC6 K80 nodes, speedup as a function of the number GPU nodes from 1 to 128 nodes scales, as expected, sub-linearly due to low task load.

We expected noticeable variation in results from run to run due to load imbalances in the computation, to resource contention for physical hardware or to nodes failing and being automatically replaced by Azure. We tested this variability using repeated runs and saw run times that varied by up to $+/-8.5 \%$ from the mean.

The gigapixel pilot results are broadly as expected with the system performing well up to the point where the amount of work available is no longer enough to keep each node fully busy. It therefore failed the GustafsonBarsis' scaling law as the computational demand was not a good match to the compute resources available. With this pilot of the system architecture complete we moved on to evaluate the full terapixel computation.

The terapixel experiment was more challenging, requiring substantially more compute time and output storage. For these reasons the smallest number of nodes we evaluated the terapixel computation with was 64 , and even with 64 GPUs the total run time was approximately 45 hours. In contrast to the gigapixel image we predicted the results from the terapixel experiment should exhibit close to linear speedup as the number of compute nodes scales from 64 to 128 nodes because the amount of work in the terapixel computation easily exceeds the quantity of compute resource available.

The measured scaling results for the terapixel image are shown in Fig. 5. These show that the system is scaling better than linearly, i.e. as more compute nodes are added the system is running faster than we would predict.

Investigating this result in detail we found a small number of outlier tasks had affected some runs. These outliers ran for 70 times longer on average than we would have expected. Further investigation of the task performance metrics uncovered that a small number of rendering tasks were running without GPU acceleration in Blender. This was not a consistent proportion on every run
TABLE 4

Terapixel Normalised Performance Data (K80)

\begin{tabular}{|c|c|c|c|}
\hline Run & $\begin{array}{l}\text { Time lost to } \\
\text { outlier tasks (s) }\end{array}$ & $\begin{array}{l}\text { Lost nodes } \\
\text { (equivalent) }\end{array}$ & Normalised run time (s) \\
\hline 64 & 159305 & 1.00 & 150550 \\
\hline 80 & 49596 & 0.40 & 119815 \\
\hline 96 & 27581 & 0.27 & 98909 \\
\hline 112 & 60151 & 0.75 & 81964 \\
\hline 128 & 0 & 0.00 & 73268 \\
\hline
\end{tabular}

Outlier tasks formed a small percentage of total tasks that ran without GPU acceleration, these caused an inflated measured speedup ratio to be calculated, normalized run time and node count removed these outlier tasks from consideration.

and the effect is summarised in Table 4.

As a result of the outliers we needed a method to estimate speedup fairly, to do this we excluded outlier tasks from consideration in the scaling calculations. The effect of the outlier tasks is removed from both from the run times of nodes and from the available node GPU resource. The resulting normalised speedup is also shown in Fig. 5. This shows the speedup ratio improving close to linearly as expected, with a small variation from the ideal that is now within the measured $+/-8.5 \%$ range. In later runs we ran a script to detect and restart nodes that had long running tasks, and subsequently found a versioning issue with drivers on some nodes that we corrected at system initialization before runs began.

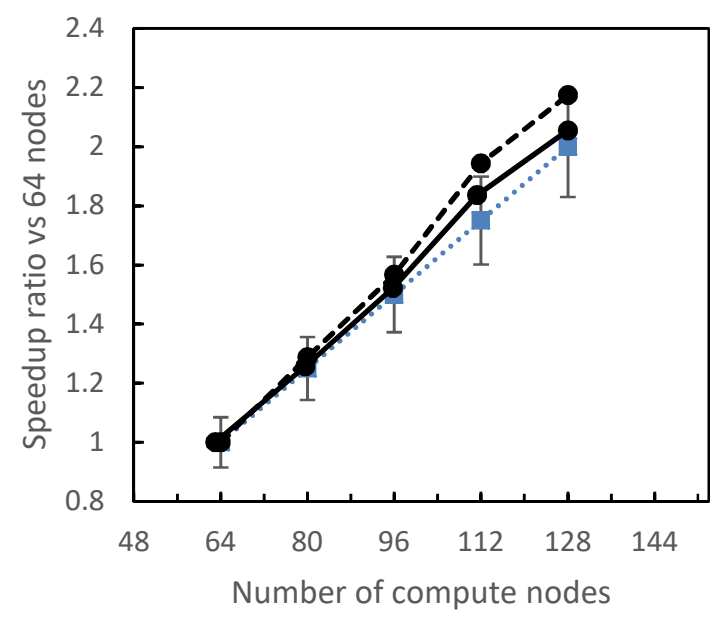

....... Ideal - Normalised

Fig. 5. Evaluating the scalability of the terapixel computation on NC6 K80 nodes, plotting speedup ratio as a function of the number of GPU nodes from 64 to 128 nodes, the bars show the $+/-8.5 \%$ range of variability we measured in repeated runs.

We found that our own metrics and those comparable metrics that were collected by Azure were in close agreement regarding run time. However, our metrics collected finer grained results from within the computation in each task allowing verification of the sub-tasks shown Fig. 2. 


\subsection{Scaling to supercomputer performance}

Following the two successful pilots we were interested to investigate the scale of computing performance the cloud could deliver for our application. Simultaneously a new GPU node became available in the Azure public cloud and over 1024 of them were available on demand. The new NC6v3 has six cores and one NVIDIA TESLA V100 GPU [29] delivering 14 teraFLOPS of single precision compute. Theoretically this would allow a system of 1024 nodes to scale to over 14 petaFLOPS compute performance, a level equivalent to systems high in the top 500 list of global supercomputers [30].

Based on a series of trial runs we found our single node performance metric database could not cope with the rate at which of metrics data being generated in these larger runs. We adjusted the architecture so that parallel data stores were used for streaming metrics data. Because of the size of terapixel image computation we started our collection of metrics at 64 nodes and scaled up to collect data for runs at 128, 256, 512, 768 and 1024 nodes.

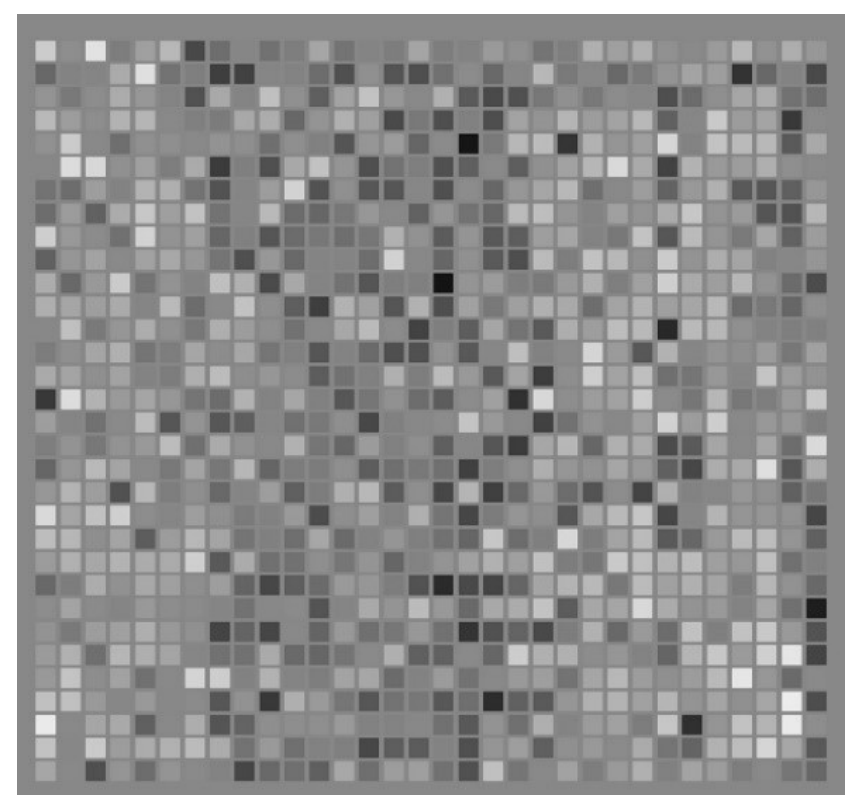

Fig. 7. Heat map of total computation time for all tasks computed by each GPU node in the 1024 run, median is $50 \%$ grey, white is nodes executing for more time and black nodes for less time, we expect the random distribution of totals shown as individual task times vary.

Recalling issues in the pilot runs with slow tasks not running on the GPU we confirmed runs were successful by analysing the spread of task run times and the sum of compute time for all tasks on each node. This time there were no outlier tasks in any of the runs. The total compute time per node is shown in Fig. 7 as a heatmap for the 1024 run. As expected, this shows a random spread of total compute time resulting from the natural variations in individual task compute times. However, the results for the 768 run revealed that four nodes had significantly lower total compute values. Analysing total compute time per node across all runs showed the same behaviour in run 256. This turned out to be a standard behavior where Azure can deallocate and then restore a small number of nodes, in both cases in groups of four. The time taken for
TABLE 5

Terapixel Normalised Performance Data (V100)

\begin{tabular}{rrrrr}
\hline & \multicolumn{1}{l}{$\begin{array}{l}\text { Run } \\
\text { Run }\end{array}$} & Time (s) & \multicolumn{1}{l}{$\begin{array}{l}\text { Lost } \\
\text { Time (s) }\end{array}$} & $\begin{array}{l}\text { Nodes } \\
\text { Normalised } \\
\text { Nodes }\end{array}$ \\
\hline \hline 64 & 45632 & 0 & 0.000 & $\mathbf{6 4 . 0}$ \\
80 & 36567 & 0 & 0.000 & $\mathbf{8 0 . 0}$ \\
96 & 30900 & 0 & 0.000 & $\mathbf{9 6 . 0}$ \\
112 & 26551 & 0 & 0.000 & $\mathbf{1 1 2 . 0}$ \\
128 & 22778 & 0 & 0.000 & $\mathbf{1 2 8 . 0}$ \\
256 & 11469 & 5467 & 0.477 & $\mathbf{2 5 5 . 5}$ \\
512 & 5726 & 0 & 0.000 & $\mathbf{5 1 2 . 0}$ \\
768 & 3824 & 5896 & 1.542 & $\mathbf{7 6 6 . 5}$ \\
1024 & 2901 & 0 & 0.000 & $\mathbf{1 0 2 4 . 0}$ \\
\hline \hline
\end{tabular}

Results from runs using 64 to 1024 NVDIA V100 GPU nodes. The final column is the normalised number of GPU nodes after accounting for nodes dropped and automatically reinstated by Azure during runs 256 and 768 .

this reallocation was approximately twenty minutes of real time, hence those nodes showed significantly lower compute time totals compared to their peers. In order to account for this behaviour we calculated the normalised number of nodes for the scaling calculations, based on the missing compute time, as shown in Table 5.

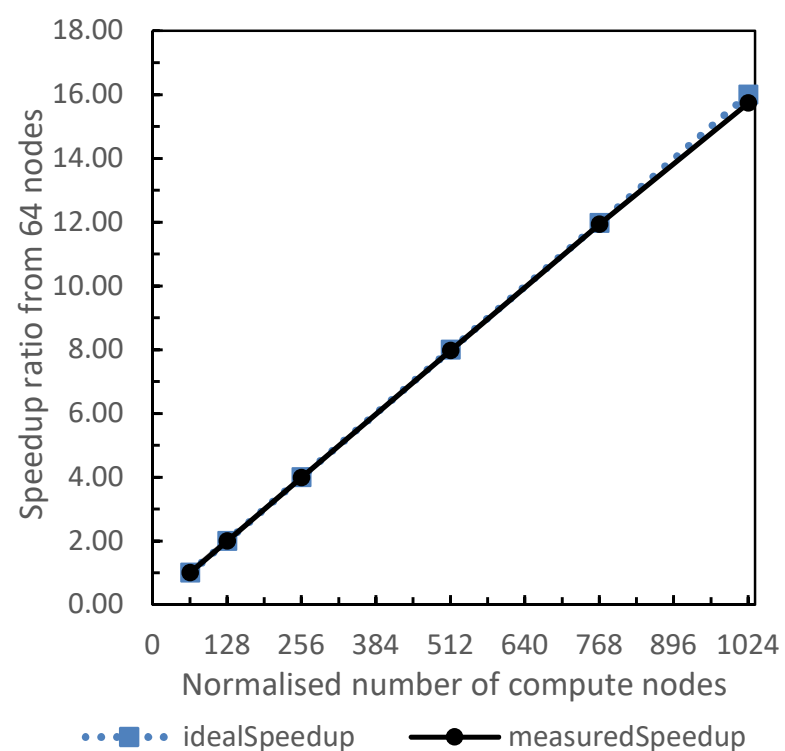

Fig. 8. The speedup ratio plotted for the terapixel computation from 64 to 1024 NC6v3 V100 GPU nodes, the speedup shows close to linear scaling achieving $98 \%$ efficiency at 1024 nodes.

Using the normalised number of nodes for each run we are able to plot the scaling graph in Fig. 8. The shows the terapixel calculation scales to make good use of from 64 to 1024 nodes with a calculated efficiency of $98 \%$, utilizing 14 of the maximum 14.3 petaFLOPS available.

An important ends-based performance measure is the wall clock time to compute an answer [18]. The total compute time for all tasks in one terapixel image varies slightly from run to run. To estimate the wall clock time to run the terapixel image on a single NC6v3 node we averaged across the total task compute time for each run, resulting 
in an estimate of 2,940,581 seconds or approximately 34 days of compute time. This compares to 45,632 seconds or 12.7 hours on 64 nodes and to 2,901 seconds or 48 minutes on 1024 nodes, as reported in Table 5.

\subsection{Accessible visualization platform}

One important outcome for us was that the terapixel visualization was accessible to users on thin client devices. The aim being to make results from our supercomputer visualizations open to a range of audiences. Serving the hierarchical image format from Azure cloud Blob store to a krpano viewer enabled web page allowed the terapixel image to be browsed on devices including 24 Mpixel video walls, desktop and laptop computers, UHD TVs, tablets, cell phones and even a gym cycling machine, as illustrated in Fig. 9. You can try it on your own device at this link [31].

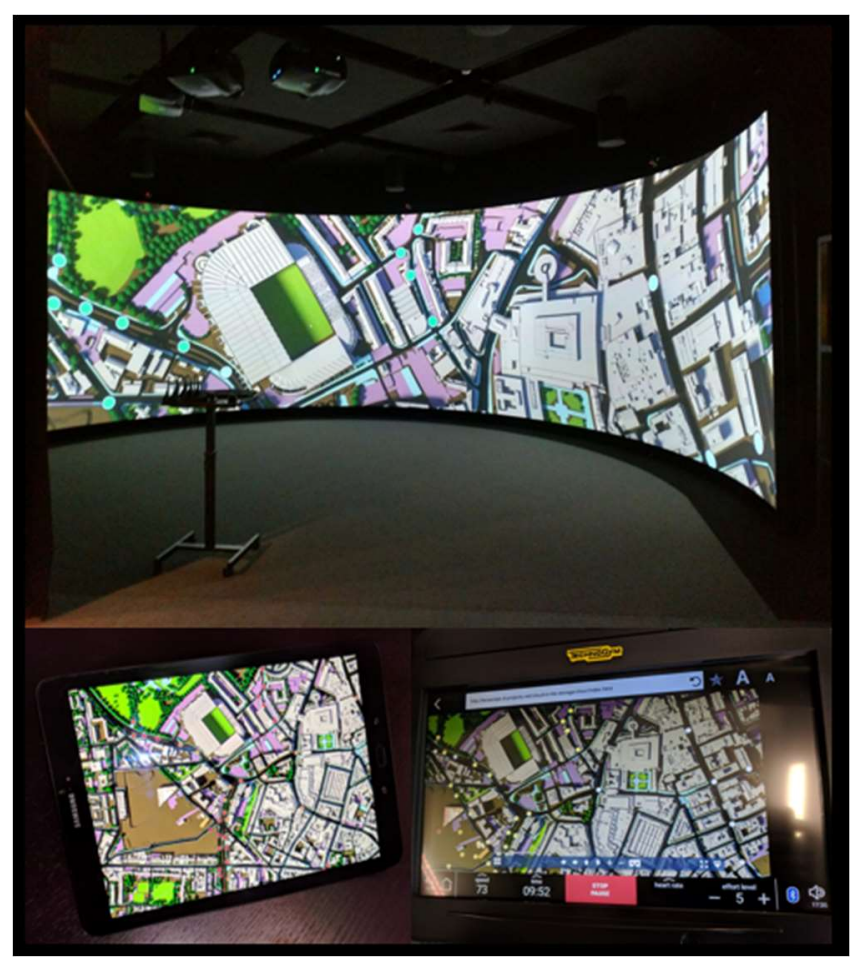

Fig. 9. The hierarchical krpano image format for serving the visualization to users from Azure Blob store allows it to be viewed interactively on a wide range of thin client devices, shown here anticlockwise from top are the 8 Mpixel Curtin HIVE cylinder, a Samsung Galaxy S3 and on our local gym cycling machine.

\section{Discussion}

\subsection{Computational performance}

The system, as reported above, did scale to use the resources available, it was not ultimately limited by Amdhal's law and was able to take advantage of Gustafson-Barsis' law application scaling to use all the available resources available efficiently.

However, this success did require software engineering resources to develop and test the system. A number of unforeseen hurdles had to be overcome with specialist help relating to remote systems issues that were difficult to access or difficult to resolve without cloud supplier in- tervention. These are likely to be teething issues with new cloud systems and compared to the procurement and systems support needed to build our own equivalent supercomputer were a low barrier to making progress.

The send-image system architecture was successful in allowing us to deliver the resulting visualization to thinclients across the whole spectrum of display types available to end-users. Accessibility of results to a wide range of stakeholders is an important goal for urban data systems.

\subsection{Application performance}

For end users it is application rather than computational performance that is of paramount importance. We consider Gustafon's [18] ends-based metrics below:

The time to compute an answer dropped from an infeasible 34 days to a reasonable 48 minutes on the largest cloud system tested. This fulfills our original end user target of producing a terapixel image once a day. High peak demand daily events such as this could be a cost-effective use case for cloud HPC, as suggested in [15], since a dedicated system would be idle for 23 hours every day.

The degree of completeness of the answer is more complex to assess because in photo-realistic graphics we can always do more. However, we have produced the first terapixel visualization of an urban digital twin and have done so with high quality 3D geometry, rendered using path tracing with a good sampling level of up to 20 rays per pixel, or a maximum of 20 trillion rays cast.

The maximum feasible problem size we measure by image resolution in pixels, the cloud enabled the production of a terapixel image that would be impractical on a desktop system. This is also the highest resolution we need for our $1.2 \mathrm{~km} \times 1.2 \mathrm{~km}$ city model as we don't have urban sensors or city geometry at sub-millimeter scale.

System reliability is critical in long or large rendering operations. The self-repairing behavior demonstrated by the cloud, even though more complex to account for in scaling calculations, is a benefit. It is something that we did not have in our private cloud [24] where days of runtime could be lost while system repairs took place.

Hardware updates from an application viewpoint the change in the cloud from NVIDIA K80 to V100 GPU nodes was transparent delivering about three times improvement in performance. This would have been a very significant procurement effort for an upgrade to a local supercomputer installation but is easily supported in the cloud.

Confidential data protection building a send-image architecture in the cloud means confidential data, particularly the 3D model of the city was protected. This helped enforce license restrictions as no model data was sent to client devices, this contrasts with webGL browser solutions where model data is sent to client devices.

\subsection{Energy use and financial cost}

On-premises supercomputers require long term commitments to energy and running costs, whereas for cloud supercomputers we can consider these as one-off costs for each run. For the terapixel runs we predict that both energy use and costs should be constant with number of nodes as the compute time falls linearly with the number 
of nodes used, as shown in Fig. 6.

We estimate total energy per run, $E$, in $\mathrm{kWh}$ as

$$
E=p_{a v} * r_{\text {norm }}
$$

Where $p_{a v}$ is the average power drawn in $\mathrm{kW}$ by the system and, $r_{\text {norm, }}$ is the normalized total run time on $n$ nodes. The results are shown in Table 6 and as expected $E$ remains approximately constant across runs.

TABLE 6

TERASCOPE SYSTEM ENERGY USE

\begin{tabular}{rrrr}
\hline $\begin{array}{c}n \\
\text { nodes } \\
\text { used }\end{array}$ & $\begin{array}{c}p_{a v} \\
\text { average pow- } \\
\text { er draw (kW) }\end{array}$ & $\begin{array}{c}r_{\text {norm }} \\
\text { normalised } \\
\text { run time (hrs) }\end{array}$ & $\begin{array}{c}E \\
\text { total power } \\
(\mathrm{kWh})\end{array}$ \\
\hline \hline 64 & 7.44 & 41.82 & 311 \\
80 & 9.47 & 33.28 & 315 \\
96 & 11.42 & 27.47 & 314 \\
112 & 13.66 & 22.77 & 311 \\
128 & 15.02 & 20.35 & 306 \\
\hline \hline
\end{tabular}

Energy use summarised per run on the NC6 K80 nodes, more nodes use more energy but run for a shorter time so total power drawn is similar across runs.

To understand our experimental costs, we similarly estimated total cost per run, $C$, as

$$
C=c_{h r} * r_{\text {norm }}
$$

Where $c_{h r}$ is the average cost per hour of the number of nodes. Table 7 shows costs stay roughly constant as expected. It is worth noting this doesn't include development time or failed runs, we estimated these factors doubled our costs for this set of experiments, but in a longer series of production runs would become less significant.

TABLE 7

TERASCOPE SYSTEM COSTS

\begin{tabular}{rrrrr}
\hline $\begin{array}{c}n \\
\text { Nodes } \\
\text { used }\end{array}$ & $\begin{array}{c}C_{h r} \\
\text { cost per } \\
\text { hour }(\mathrm{f})\end{array}$ & $\begin{array}{c}r_{\text {norm }} \\
\text { Normalised } \\
\text { run time (hrs) }\end{array}$ & $\begin{array}{c}C \\
\text { Total } \\
\text { Cost }(\mathrm{f})\end{array}$ & $\begin{array}{c}P P \\
\text { Pixels/Pound } \\
\text { (millions) }\end{array}$ \\
\hline \hline 64 & 42.94 & 41.82 & 1796 & 612 \\
80 & 53.68 & 33.28 & 1787 & 615 \\
96 & 64.42 & 27.47 & 1770 & 621 \\
112 & 75.15 & 22.77 & 1711 & 642 \\
128 & 85.89 & 20.35 & 1748 & 629 \\
\hline \hline
\end{tabular}

Costs are summarized for each run on the NC6 K80 nodes, the costs remain similar across runs because the total compute time required for the terapixel computation is constant.

A final ends-based metric from Gustfson is performance divided by system cost, which we estimate for each run as the ratio of pixels per pound spent, $P P$.

$$
P P=\frac{P i x}{C}
$$

Where Pix is one trillion, the total number of pixels in our rendered image. If our scaling is sub-linear, we will see this ratio go down and see diminishing returns on our investment. Because in the terapixel case our scaling is close to linear $P P$ is approximately constant varying by less than $5 \%$, as shown in Table 7.

\subsection{Future scaling using the cloud}

We have demonstrated the ability to scale an application on current generation GPU technology using the cloud to over 1000x the performance of a single desktop system. As application researchers this future scaling allows us to experiment with creating the software tools that we need for the next generation of visualization applications. How far into the future this allows us to plan depends on predicting how well system performance might improve.

The future of Moore's law is being challenged and scaling out to the cloud has been suggested as one way to mitigate the need for continuous hardware improvement. However, while we have demonstrated the cloud can provide huge advantage during any single hardware cycle it, at best, scales up linearly. Over time this could not match the exponential scaling Moore's law has delivered. Even if datacenters could scale exponentially by floor area, so simultaneaously so would the power and cooling needs.

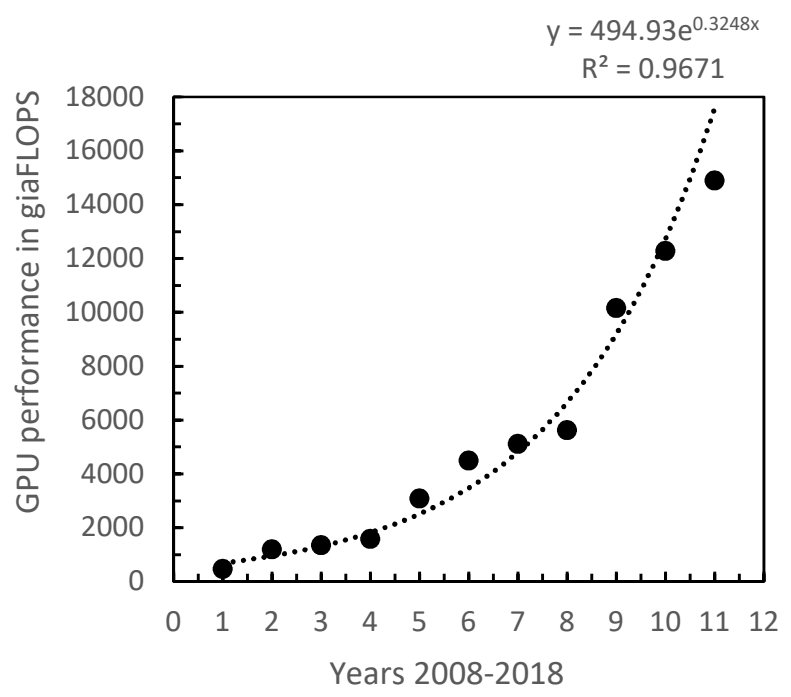

Fig. 10. The performance in gigaFLOPS of GPU devices from NVIDIA since 2008, an exponential regression fits this well allowing prediction of future performance if the same rate of improvement continues.

Fortunately for GPU system users a range of improvements mean the whole software/hardware stack for graphics and Al continues to demonstrate exponential growth [32], as illustrated in Fig. 10. If we use NVIDIA's GPU performance to date as a guide and predict forward, then it should take about twenty years for the petaFLOP performance we have demonstrated in this paper to become the norm on everyday desktop computers. Technologies already announced that will contribute to this include the NVIDIA RTX hardware support for ray tracing [33] and the Blender 2.8 EEVEE algorithms [34]. EEVEE provides close to path traced image quality at two hundred times the speed of the standard Cycles renderer. 


\section{Conclusions}

We set out to address three goals:

Design a new supercomputer application architecture for scalable visualization using the public cloud. We delivered this using Azure APIs, the krpano viewer and our own task generation, compute and metric monitoring codes.

Produce the first terapixel urban loT visualization supporting live daily updates. We exceeded this target and demonstrated we can render these as frequently as every hour at the same cost as a single node that would take 34 days and deliver the result to accessible thin clients.

Undertake a rigorous performance evaluation of cloud supercomputing for visualization applications. We have demonstrated Gustafson-Barsis' scaling holds for terapixel visualization and achieved twenty sample path traced rendering rates that are equivalent to a real time frame rate of $200 \mathrm{~Hz}$ for path tracing full HD images.

Four key lessons from the terapixel project have been:

- The cloud can deliver world class supercomputing performance (to 14petaFLOPS using 1024 V100 GPUs) at reasonable cost. This enabled us to undertake research that would not have been feasible on any publically available systems in the UK.

- Cloud systems upgrade quickly, the GPU upgrades in the duration of this project significantly improved performance transparently to the application code.

- Monitoring and verification are essential to be sure what the cloud system is doing, when and to confirm computations at scale are completed correctly from an application viewpoint.

- The access to future scale hardware at relatively low cost allows us to research new ideas that are well beyond the scope of current everday systems.

For the future we could use the computational power of the system in several different ways, e.g. to produce daylight correct images of the city hourly throughout the day, to produce a lightfield rendering which would allow soft focus effects in real time without re-rendering and/or deliver lightfield VR/AR display support.

We don't need more pixels in the current geographic area but a larger digital twin covering a wider area around Newcastle-upon-Tyne would be a benefit for the region. For example, a $33 \mathrm{~km}^{2}$ map would require a petapixel image that would take 33 days rendering on 1024 nodes, setting a future challenge for our cloud architecture.

Finally, based on our experience, we would support the conclusions in [15] that future cloud supercomputing projects would benefit from systems improvements including: better metrics collection, performance visualization modules, transparent error reporting and simpler purchasing, licensing and cost tracking systems.

\section{ACKNOWLEDGEMENTS}

With thanks to; Northumbria VRV Studio for the VNG 3D model of Newcastle. The National Innovation Centre for Data and their local partners Newcastle City Council, for Azure cloud time. Microsoft UK for invaluable guidance in using the Azure at supercomputer scale. The Alan Turing Institute, London for supporting the project under EPSRC grant EP/N510129/1 and Nicolas Holliman's Turing Fellowship. The EPSRC UKRIC project for funding and supporting the Newcastle Urban Observatory. Siemens UK, in Poole sponsoring Manu Antony's EPSRC iCASE award.

\section{REFERENCES}

[1] P. James, R. Dawson, N. Harris, and J. Joncyzk, "Urban Observatory Environment Data." Newcastle-upon-Tyne, 2014.

[2] E. W. Bethel, H. Childs, and C. Hansen, High Performance Visualization: Enabling Extreme-Scale Scientific Insight. CRC Press, 2012.

[3] Reinfeld Klaus, "krpano.com," krpano Gesellschaft mbH. [Online]. Available: https://krpano.com/. [Accessed: 02-Jan2019].

[4] Blender Online Community, "Blender 2.79 Reference Manual - Blender Manual." [Online]. Available: https://docs.blender.org/manual/en/latest/. [Accessed: 02Jan-2019].

[5] N. Ferreira, J. Poco, H. T. Vo, J. Freire, and C. T. Silva, "Visual exploration of big spatio-temporal urban data: A study of new york city taxi trips," IEEE Trans. Vis. Comput. Graph., vol. 19, no. 12, pp. 2149-2158, 2013.

[6] M. Batty, "Digital twins." SAGE Publications Sage UK: London, England, 2018.

[7] Centre for Digital Built Britain, "The Gemini Principles." Centre for Digital Built Britain and Digital Framework Task Group, Cambridge, 2018.

[8] G. Boulton et al., "Science as an open enterprise," $R$. Soc., 2012

[9] A. Cairo, The truthful art: Data, charts, and maps for communication. New Riders, 2016.

[10] D. Agarwal et al., "Data-intensive science: The Terapixel and MODISAzure projects," Int. J. High Perform. Comput. Appl., vol. 25, no. 3, pp. 304-316, 2011.

[11] Y. Feng et al., "Terapixel imaging of cosmological simulations," Astrophys. J. Suppl. Ser., vol. 197, no. 2, p. 18, 2011.

[12] P. Rosenfield et al., "AAS WorldWide Telescope: A Seamless, Cross-platform Data Visualization Engine for Astronomy Research, Education, and Democratizing Data," Astrophys. J. Suppl. Ser., vol. 236, no. 1, p. 22, 2018.

[13] Microsoft Azure, "Azure Windows VM sizes," 2018. [Online]. Available: https://docs.microsoft.com/en-us/azure/virtualmachines/windows/sizes-general. [Accessed: 02-Jan-2019].

[14] AWS, "Amazon Elastic Graphics - Features." [Online]. Available: https://aws.amazon.com/ec2/elasticgraphics/features/. [Accessed: 02-Jan-2019].

[15] M. A. S. Netto, R. N. Calheiros, E. R. Rodrigues, R. L. F. Cunha, and R. Buyya, "HPC cloud for scientific and business applications: taxonomy, vision, and research challenges," ACM Comput. Surv., vol. 51, no. 1, p. 8, 2018.

[16] G. M. Amdahl, "Validity of the single processor approach to achieving large scale computing capabilities," in Proceedings of the April 18-20, 1967, spring joint computer conference, 1967, pp. 483-485.

[17] J. L. Gustafson, "Reevaluating Amdahl's law," Commun. ACM, 
vol. 31, no. 5, pp. 532-533, 1988.

[18] J. Gustafson, "Teraflops and other false goals," IEEE Paralle/ Distrib. Technol. Syst. Appl., vol. 2, no. 2, pp. 5-6, 1994.

[19] N. S. Holliman, C.-M. Wang, and P. M. Dew, "Mistral-3: parallel solid modelling," Vis. Comput., vol. 9, no. 7, pp. 356370, 1993.

[20] N. Holliman and P. Watson, "Scalable Real-Time Visualization Using the Cloud," IEEE Cloud Comput., vol. 2, no. 6, pp. 90-96, 2015.

[21] J. Chen, I. Yoon, and W. Bethel, "Interactive, internet delivery of visualization via structured prerendered multiresolution imagery," IEEE Trans. Vis. Comput. Graph., vol. 14, no. 2, pp. 302-312, 2008.

[22] Intel, "Rethinking Visual Cloud Services for Evolving Media." Intel Corporation, 2019.

[23] E. H. Adelson, C. H. Anderson, J. R. Bergen, P. J. Burt, and J. M. Ogden, "Pyramid methods in image processing," $R C A$ Eng., vol. 29, no. 6, pp. 33-41, 1984.

[24] S. Dowsland, M. Turner, and N. Holliman, "A Scalable Platform for Visualization Using the Cloud," in International Conference on Computer Games, Multimedia \& Allied Technology (CGAT). Proceedings, 2017, p. 54.

[25] Red Hat, "How Ansible Works | Ansible.com." [Online]. Available: https://www.ansible.com/overview/how-ansibleworks. [Accessed: 02-Jan-2019].

[26] Microsoft, "Overview of Batch APIs and tools," docs.microsoft.com, 2018. [Online]. Available: https://docs.microsoft.com/en-us/azure/batch/batch-apistools. [Accessed: 27-Jan-2019].

[27] Elasticsearch B.V., "Elastic Stack and Product Documentation," 2019. [Online]. Available: https://www.elastic.co/guide/index.html. [Accessed: 27-Jan2019].

[28] NVIDIA, "NVIDIA $®$ TESLA $®$ GPU ACCELERATORS The Tesla family of GPU Accelerators includes: Tesla K80 GPU Accelerator," 2014.

[29] NVIDIA, "TESLA V100 Performance Guide." 2018.

[30] E. Strohmaier, J. Dongarra, H. Simon, and M. Meuer, "November 2018 | TOP500 Supercomputer Sites," 2018. [Online]. Available: https://www.top500.org/lists/2018/11/. [Accessed: 31-Jan-2019].

[31] N. Holliman, M. Antony, S. Dowsland, and M. Turner, "Terascope," 2018. [Online]. Available: http://terascope.diprojects.net/cloudviz-tile-storage-1024/vtour/index.html. [Accessed: 12-Feb-2019].

[32] Jensen Huang, "Keynote NVIDIA's 2018 GPU Technology Conference | The Official NVIDIA Blog," 2018. [Online]. Available: https://blogs.nvidia.com/blog/2018/03/26/livejensen-huang-keynote-2018-gtc/. [Accessed: 10-Feb-2019].

[33] NVIDIA, "TURING GPU ARCHITECTURE." 2018.

[34] Blender Foundation, "Blender 2.8 - blender.org," 2018. [Online]. Available: https://www.blender.org/2-8/\#eevee. [Accessed: 03-Feb-2019].

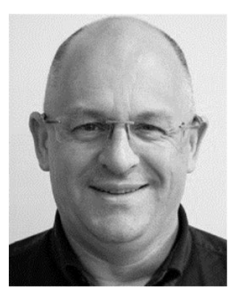

Nicolas S. Holliman Ph.D. (University of Leeds 1990) Computer Science, B.Sc. (University of Durham, 1986) joint honours Computing with Electronics. He worked as a Researcher at Lightwork Design Ltd., was Principal Researcher at Sharp Laboratories of Europe Ltd, Reader in Computer Science at Durham University and Professor of Interactive Media at the University of York. He is currently Professor of Visualization at Newcastle University and a Fellow of The Alan Turing Institute, London. He has published articles and patents in visualization, human vision, computer vision, parallel computing and $3 \mathrm{D}$ displays. He is a member of the IS\&T, the ACM, a fellow of the Royal Statistical Society and a member of the IEEE Computer Society.

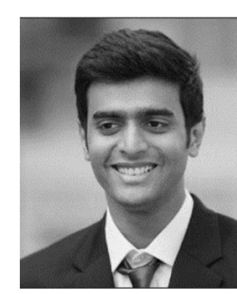

Manu Antony B.Eng. Electrical, Electronics and Computer Engineering (Newcastle University 2014). Since 2016 he has been an EPSRC iCASE award PhD student in data visualization sponsored by Siemens Ltd, Poole, UK. He has worked both in the tech industry and the finance industry.

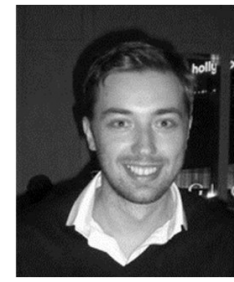

James Charlton Ph.D. (Northumbria University, 2011), Architectural Technology B.Sc. (Northumbria University, 2006). He has worked as a Research Assistant, Research Fellow and is now a Senior Lecturer in Architecture at Northumbria University, heading up the VRV Studio and VNG city model. James has published articles in; digital visualisation, virtual city modelling, performance analysis and urban design.

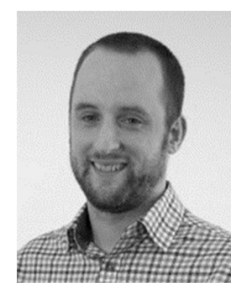

Stephen Dowsland M.Sc. (Newcastle University 2009) Town Planning, B.A. Hons (Northumbria University 2007) Geography. He worked in local government before specialising in mapping and front-end web development at British Airways as part of the major incident planning team. He joined Newcastle University as a Research Software Developer specialising in scalable cloud architectures. He now works at the National Innovation Centre for Data at Newcastle as a Senior Software Specialist working on scalable cloud and big data processing.

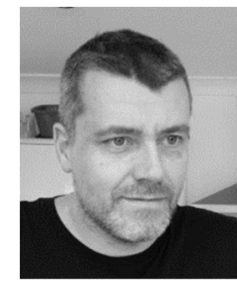

Phil James B.A.(Newcastle) is a Senior Lecturer in Engineering. He is currently director of the Newcastle Urban Observatory and co-leads the UK National Observatory Programme. He manages and directs the observatory programme generating strategic partnerships with researchers, civic society and industry. His research is at the intersection of Engineering and Computer Science with a recent focus on loT and environmental monitoring and how we apply emerging technologies to realworld solutions. He is PI on the EPSRC CORONA (City Observatory Research platfOrm for iNnovation and Analytics) project and participates as Co-I in a research portfolio of interdisciplinary research worth over $£ 15 \mathrm{~m}$. He is a Fellow of the Royal Geographical Society.

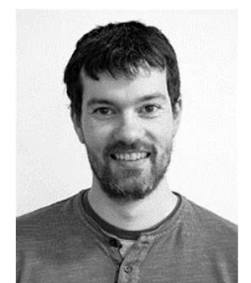

Mark Turner M.Sc. (Newcastle University 2012), B.Sc. in Computing (Northumbria University 2008) leads the Research Software Engineering team at Newcastle. The team focuses on delivering software engineering expertise for research projects across the university. In 2016 he was elected as a trustee for the UK Research Software Engineering Association. Since joining the Newcastle in 2012 he has designed and implemented software applications for research projects including the gamification of stroke rehabilitation physical therapy, mobile applications for alerting stakeholders to damage to rock art carvings and supercomputer scale cloud computing. 